\title{
The reinstatement effect: Amount of noncontingent reward in the runway'
}

PATRICK E. CAMPBELL, Southern Methodist University, Dallas, Tex. 75222, DEAN L. FIXSEN, and ELERY PHILLIPS, Bureau of Child Research and The University of Kansas, Lawrence, Kansas 66044

The effects of a noncontingent feeding procedure following extinction of a runway response were investigated with 24 rats as Ss. Following acquisition and extinction training, four groups were formed and individually placed into the goal box and fed $0,5,10$, or 20 food pellets on each of 4 days. The effects of this procedure were tested by again running the $S s$ to an empty goal box. Running speeds were reinstated for each of the fed groups and the speed of running varied with reward magnitude. These data were discussed in terms of incentive theory and Capaldi's aftereffects hypothesis.

Reintroduction of reward following extinction of an operant response can reinstate that response even when the reward is noncontingent. This effect has been reported with rats (Campbell, Phillips, Fixsen, \& Crumbaugh, 1968), retarded children (Spradlin, Giradeau, \& Hom, 1966) and with pigeons and college students (Reid, 1957). With the exception of the Campbell et al (1968) paper these authors have interpreted these data as a matter of discrimination between acquisition and extinction conditions. Reintroducing reward after a period of extinction would, according to this view, reinstate a portion of the stimulus complex conditioned to the response during acquisition. Any resumption of responding then would be due to the associative control of the response by the reward-correlated stimuli.

Campbell et al (1968) acknowledged the possibility of associative principles accounting for this effect but proposed that incentive motivational factors could account for these data with equal ease. It is assumed that extinction is at least partially due to a decrease in incentive motivation (perhaps because of extinction of the rg-sg mechanism) and that reintroducing reward into the situation occasions the conditions for reacquisition of the rg-sg mechanism and hence renewed incentive motivational support for the response. Following Spence's (1956) incentive theory, the degree of incentive motivation should be a function of the magnitude of reward present during the reinstatement phase. The purpose of this experiment was to test this implication of Spence's theory within the reinstatement paradigm.

\section{METHOD}

Twenty-four male, naive, albino rats, approximately 70 days of age served as Ss.

The apparatus, a Hunter small-animal runway painted flat black, was $32 \times 3.5 \times 4$ in. The start and goal boxes were $12 \times 5.2 \times 4.5$ in. Two photoelectric relays located 6 in. from the beginning and end of the runway were connected to microtimers and served as start and run time measures.

Pretraining consisted of 13 days of handling and group exploration in the runway. A $23.5-\mathrm{h}$ deprivation schedule remained in effect throughout the experiment. Acquisition training consisted of 10 days of one-trial-a-day reinforced running trials. The reward was five $.097-\mathrm{g}$ Noyes pellets for all Ss. During extinction the Ss were run five trials a day for the first 6 days and one trial a day thereafter. On days when multiple trials were given the intertrial interval was approximately $20 \mathrm{~min}$. After 14 days of extinction, the Ss' median running times had stabilized at a level similar to the operant level recorded for the first day of acquisition.

The Ss were then individually placed directly into the goal box one trial a day for four days according to the following design: Group E5 was placed in the goal box and fed five $.097-\mathrm{g}$ pellets, Group E10 was fed 10 pellets, and E20 received 20 pellets. Group $C$ spent an equal amount of time in the unbaited goal box. All Ss were left in the goal box for $4 \mathrm{~min}$, the time necessary for the slowest $S$ in Group E20 to consume all 20 pellets on the first day of this procedure. The Ss were then tested for noncontingent-reward effects by again running to an unbaited goal box one trial a day for six days.

\section{RESULTS AND DISCUSSION}

Both start and run time scores were converted to reciprocals. Only the results of the run measure will be presented here since both measures gave similar results. A one-way analysis of variance on the last day's extinction data showed that the groups did not differ significantly on either response measure $(p>.05)$. The mean test phase performance for each group was as follows: $\mathrm{C}=6.25, \mathrm{E} 5=8.75$, $\mathrm{E} 10=8.95$, and $\mathrm{E} 20=11.11$. An overall $\mathrm{F}$ test revealed these means to be different $(F=3.22, \quad d f=3 / 20, \quad p<.05)$. Subsequent orthogonal contrasts showed $\mathrm{C}$ to be slower than the average of the E groups and E20 was faster than the average of $\mathrm{E} 5$ and $\mathrm{E} 10$ which did not differ significantly.

It is apparent from these data that noncontingent reward served to increase subsequent running speeds beyond what could be expected on the basis of spontaneous recovery alone. In fact, E20 Ss actually reached speeds comparable to their terminal acquisition speed. Furthermore, the superiority of E20 Ss over E5 Ss would also suggest the need for more than a purely associative theory of the reinstatement effect. If a return to the acquisition stimuli was the only determinant of reinstatement then E5 Ss would have shown greater reinstatement than the remaining groups. This prediction would follow since five pellets would reinstate the exact stimulus involved in acquisition. The 10 - and 20-pellet conditions would occasion increasing amounts of generalization decrement with the result that E20 should have shown the least amount of reinstatement. Incentive theory, however, would predict the opposite which, of course, is more in line with the present results.

Although Spence's (1956) incentive theory can account for most of the present data, the finding of equal reinstatement for the 5- and 10-pellet groups does not fit the theory. These results, including the comparability of 5 and 10 pellets, can be predicted from Capaldi's (1967) aftereffects hypothesis. Capaldi's theory would predict reinstatement effects as a function of both associative and motivational factors. According to this theory noncontingent reward would reinstate the stimulus aftereffects of reward and occasion an increase in incentive motivation. The fact that E20 was superior to E5 but E10 was not could be accounted for as follows: E5 would result in the greatest associative control but the smallest increase in incentive; E10 would suffer from generalization decrement but occasion somewhat more incentive, perhaps enough to balance the effects of greater associative control for E5; E20 would also introduce generalization decrement but the increase in incentive would be sufficiently great to outweigh the generalization. The relative contribution of associative and motivational factors 
could be easily assessed by factorially varying reward magnitude in both the acquisition and reinstatement phases.

\section{REFERENCES}

CAMPBELl, P. E., PHILlIPS, E. L., FIXSEN, D. L., \& CRUMBAUGH C. Free operant response reinstatement during extinction and time-contingent (DRO) reward. Psychological Reports, 1968, 22, 563-569.

CAPALDI, E. J. A sequential hypothesis of instrumental learning. In $K$. W. Spence and J. T. Spence (Eds.), The psychology of learning and motivation. New York: Academic Press, 1967.
REID, R. L. The role of the reinforcer as a stimulus. British Journal of Psychology, 1957, 49, 202-209.

SPENCE, K. W. Behavior theory and conditioning. New Haven: Yale University Press, 1956.

SPRADLIN, J. E., GIRADEAU, F. L., HOM, G. L. Stimulus properties or reinforcement during extinction of a free operant response. Journal of Experimental Child Psychology, 1966, 4, 369-380.

\section{NOTE}

1. This research was partially supported by Research Grant 8835 from the National Science Foundation awarded to the senior author by Southern Methodist University and by Grant ND-05362 from NINDB to the Bureau of Child Research of the University of Kansas.
Table 1

Responses in Extinction after Different Numbers of Priming Trials

\begin{tabular}{|c|c|c|c|c|}
\hline \multirow{2}{*}{$\begin{array}{r}\text { Number of } \\
\text { Priming Trials }\end{array}$} & \multicolumn{4}{|c|}{$\begin{array}{l}\text { Numbers of responses in extinction } \\
\text { Replication }\end{array}$} \\
\hline & 1 & 2 & 3 & 4 \\
\hline 5 & 34 & 0 & 1 & 1 \\
\hline 30 & 68 & 9 & 165 & 54 \\
\hline 55 & 24 & 21 & 39 & 14 \\
\hline 105 & 6 & 0 & 0 & 0 \\
\hline 205 & 0 & 46 & 17 & 68 \\
\hline 305 & 12 & 45 & 65 & 34 \\
\hline
\end{tabular}

trials ended after a minimum of 20 or after six consecutive failures to respond.

A subexperiment with six birds, one allocated to each number of priming trials was carried out first, then replicated, and replicated again with apparatus that differed in detail, within the specification given above. In a final replication, birds were given extra magazine training in inverse proportion to the number of priming trials to be given, so that all of them had eaten the same amount of food in the experimental chamber before extinction started.

\section{RESULTS}

All birds responded to the priming stimulus, 23 starting on the first exposure and one on the second exposure. Responding continued up to the limit of 355 trials of priming with some decrease in latency.

The four separate sets of birds all produced comparable numbers of responses in extinction showing the same pattern of distribution according to numbers of priming trials, i.e., an early peak, a trough, and a rise with increasing exposure to the priming procedure. This is the pattern displayed in Fig. 1 in which each data point gives the averaged performance of four birds, one from each set. Of the 24 birds that were tested, 5 gave no responses in extinction.

\section{DISCUSSION}

For practical guidance in the use of priming as a training technique, the results do not indicate that any advantage is gained by giving more than 30 primed and reinforced trials. Observations of behavior suggested that the sudden removal of the priming stimulus disturbed some of the birds in this page 227)

experiment. An improved technique, which could be fully automated, would be to use projected transparencies of food and to fade out the priming stimulus over a number of trials.

The relationship between trials and extinction scores appears to be irregular, but the shape of the curve presented in Fig. 1 corresponds with the summary given by Razran (1949) of the results of experiments on stimulus generalization in Pavlov's laboratory, i.e., "CR generalization increases in the very initial stages of training the $C R$, but upon further training begins to decrease slowly, while after a large number of reinforcements it may increase again." In the case of the present experiment there is a complicating factor which may have served to exaggerate the effect. The pecking response changes during priming trials. At first it is always directed at the food and later it may drift to a focus at the edge of the key, or become variable over its surface. The opening of the beak tends also to be reduced, suggesting that the in accessible food is first responded to as food, but later loses this special characteristic and functions as an ordinary discriminative stimulus. It may be that the "food" peck is more resistant to extinction and this factor contributes to the peak performance obtained after 30 priming trials. Further investigations are in progress.

\section{REFERENCES}

FERSTER, C. B., \& SKINNER, B. F. Schedules of reinforcement. New York: Appleton-Century-Crofts, 1957.

KONORSKI, J. Conditioned reflexes and neuron organization. Cambridge: Cambridge University Press, 1948.

RAZRAN, G, Stimulus generalization of conditioned responses. Psychological Bulletin, 1949, 46, 337-365.

SKINNER, B. F. Teaching machines. Science, 1958, 128, 969-977.

SKINNER, B. F. The technology of teaching. New York: AppletonCentury-Crofts, 1968.

TERRACE, H. S. Errorless transfer of a discrimination across two continua. Journal of the Experimental Analysis of Behavior, 1963,6, 223-232.

THORNDIKE, E. L. The psychology of learning. New York: Teachers College, 1913.

NOTE

1. This research was supported in part by the Medical Research Council of Great Britain.

2. "Prompting" and "priming" are distinguished by Skinner (1968). In "prompting," a supplementary stimulus is used "to encourage a prompt appearance of behavior which already exists in some strength," whereas in "priming" the stimulus is fully adequate to evoke the behavior for the first time. 